\title{
Operating Task Redistribution in Hyperconverged Networks
}

\author{
Mohammad Alhihi ${ }^{1}$, Mohammad Reza Khosravi ${ }^{2}$ \\ ${ }^{1}$ Departement of Communications and Electronic Engineering, Philadelphia University, Amman, Jordan \\ ${ }^{2}$ Departement of Electrical and Electronic Engineering, Shiraz University of Technology, Shiraz, Iran \\ ${ }^{2}$ Computer Engineering Departement, School of Engineering, Persian Gulf University, Bushehr, Iran
}

\begin{tabular}{l} 
Article Info \\
\hline Article history: \\
Received Feb 9, 2018 \\
Revised May 12, 2018 \\
Accepted May 20, 2018 \\
\hline Keyword: \\
Distributed processing \\
E-learning \\
Penalty \\
Redistribution \\
hyperconvergence
\end{tabular}

hyperconvergence

\begin{abstract}
In this article, a searching method for the rational task distribution through the nodes of a hyperconverged network is presented in which it provides the rational distribution of task sets towards a better performance. With using new subsettings related to distribution of nodes in the network based on distributed processing, we can minimize average packet delay. The distribution quality is provided with using a special objective function considering the penalties in the case of having delays. This process is considered in order to create the balanced delivery systems. The initial redistribution is determined based on the minimum penalty. After performing a cycle (iteration) of redistribution in order to have the appropriate task distribution, a potential system is formed for functional optimization. In each cycle of the redistribution, a rule for optimizing contour search is used. Thus, the obtained task distribution, including the appeared failure and success, will be rational and can decrease the average packet delay in the hyperconverged networks. The effectiveness of our proposed method is evaluated by using the model of hyperconverged support system of the university E-learning provided by V.N. Karazin Kharkiv National University. The simulation results based on the model clearly confirm the acceptable and better performance of our approach in comparison to the classical approach of task distribution.
\end{abstract}

Copyright (c) 2018 Institute of Advanced Engineering and Science. All rights reserved.

\section{Corresponding Author:}

Mohammad Alhihi,

Departement of Communications and Electronic Engineering,

Philadelphia University,

Amman, Jordan.

Email: malhihi@philadelphia.edu.jo

\section{INTRODUCTION}

At the present age of information technology (IT) market, the distributed or cloud-based data communication platforms are step by step replaced by the converged and hyperconverged platforms such as like software defined networks (SDNs) [1]. The infrastructures are formed over a convergent platform; so with consideration of a huge quota of the memory, the computational network resources have been previously adapted for work in data center [2]; and under a hyperconverged infrastructures, the computational capacities, the storages, the servers' facilities, and other things of networks are integrated by a software platform like SDNs. The control tool for such new platforms is provided through a general administrative console [3]. Under having the hyperconverged structure for system's resource allocation and control, it is enough that only a network/system administrator or a limited number of administrators as administrative group are to be placed in a site. It thus waives the additional costs for the maintenance of data communication system. Thus, this platform is one of the best alternatives for the organization systems in the world.

In this current work, we consider systems along with several users with the independent territorial location which it is towards the increase of the number of requests periodically, for example, the service- 
based system in the university E-learning model [4], [5]. As follows, we review some existing approaches in the field of study. The unexpected situations in the problem with hardware and software system support negatively affect on the performance of the system. So, a good solution should consider such situations of the hyperconverged server in which network must quickly redistribute the available resources in order to decrease the negative impact of these problem situations. In this regard, the problem of the operating redistribution of the resources has been stated in [6]-[9]. For example in them, there is a proposed method based on mathematical model of the operating control system which permits us to consider the requirements for operativity of the decision making with concerning a redistribution of the resources and the information incompleteness. Another model is based on the multilevel tree of the hyperconverged structure description.

A goal of us in this research is to prove the objective function for search problem of the rational redistribution in task sets. We also propose a new quality distribution criterion for the problem. The paper is organized as follows. The basic concepts about the proposed work are stated in the second section whereas the third and fourth sections represent all details of the work. The fifth section includes numerical results and the final section is the conclusions.

\section{FUNDAMENTALS OF THE PROPOSED METHOD}

Using a new approach towards the explained research objective let us to define and solve the problem. As stated, the operating task redistribution through nodes of a hyperconverged network must be solved by a new approach based on distributed processing. For solving the problem, it is necessary to find the existing breakings of the task sets. The problem is solved at the network level where the average packet delay at the network becomes the lowest value. The main aim is development of a method for search in the rational task redistribution processes via the nodes of network. In the next two sections, we firstly formulate the task redistribution problem; then, we solve it.

\section{THE TASK FORMULIZATION}

The objective function for computing quality coefficient of rational breaking in task sets $(Z)$ is determined by Equation (1) when we have the nodes $y_{a} \in Y$.

$$
F^{(\gamma)}=\frac{1}{u_{z, \max }} \cdot \sum_{b=1}^{h_{z}} \sum_{a=1}^{h_{y}} m_{z_{b, a}} \cdot s_{z_{b, a}}
$$

where $u_{z, \max }$ is the maximum value of task sets which is independent from the distribution $\gamma$ for determining the maximum (general) intensity of the task exchange with the nodes of the network according to the relation of $u_{z, \max }=\sum_{b=1}^{h_{z}} \sum_{i=1}^{h_{y}} u_{z_{b, i}} . h_{z}$ is the number of the tasks under process at the network. $h_{y}$ is the number of existing nodes in the network. $u_{z_{b, i}}$ is the intensity of the task exchange $\left(Z_{B} \in Z\right)$ with the node $Y_{I} \in Y . \quad m_{z_{b, a}}$ is the required computational resource of the node $Y_{A} \in Y$ which is necessary for subtask execution towards $Z_{B} \in Z$. $s_{z_{b, a}}$ is the penalty during subtask distribution of the task $Z_{B}$ to the node $Y_{A}$ which is determined by the relation of $s_{z_{b, a}}=\sum_{i=1}^{h_{y}}\left(u_{z_{b, i}} \cdot h_{w a, i}\right) / \phi_{z_{b}} \cdot h_{w a, i}$ is the length of the quick-step between the nodes $y_{a}$ and $y_{i}$, determined by the data transmission channels which are the part of this path. $\phi_{z_{b}}$ is the required computational resource for the task processing.

The required distribution $\gamma$ must be satisfied for the following conditions [3] where $\phi_{y_{a}}$ is the available computational resource of the node $Y_{A}$.
1) $\forall y_{a} \in Y \mid \sum_{b=1}^{h_{z}} m_{z_{b, a}} \leq \phi_{y_{a}}$;
2) $\forall z_{b} \in Z \mid \sum_{a=1}^{h_{y}} m_{z_{b, a}} \leq \phi_{z_{b}}$; 
3) $\sum_{a=1}^{h_{y}} \phi_{y_{a}} \geq \sum_{b=1}^{h_{z}} \phi_{z_{b}}$;

4) $s_{z_{b, a}} \geq 0, m_{z_{b, a}} \geq 0 ; 1 \leq a \leq h_{y}, 1 \leq b \leq h_{z}$

Under the mentioned conditions, the task is processed at the network where it can be formulated as follows. For the case which the task set $Z$ is assigned to the nodes $Y$, it is determined by the tuples $\left\langle Z, \phi_{z}, U_{z}\right\rangle$ and $\left\langle Y, \phi_{y}, H_{w}\right\rangle$; where $\phi_{z}=\left(\phi_{z_{1}}, \ldots, \phi_{z_{h_{z}}}\right)$ is the vector of the required computational resources for sets processing of the task, $U_{z}=\left\|u_{z_{b, i}}\right\|$ is the matrix of the intensity of the exchange of task sets $Z$ with the nodes of $Y, \phi_{y}=\left(\phi_{y_{1}}, \ldots, \phi_{y_{h_{y}}}\right)$ is the vector of the available computational resources, and $H_{w}=\left\|h_{w_{a, i}}\right\|$ is the matrix of the lengths of the quick-steps between each pair of node in the network in which for $y_{a}$ and $y_{i}$, we have the conditions of $1 \leq a \leq h_{y}$ and $1 \leq i \leq h_{y}$.

It is necessary to find the distribution $\gamma$, which satisfies the conditions 1 to 4 , above-mentioned, to do so, the Equation (1) gives us the lowest value. For constructing the solution algorithm of this defined problem, it is convenient to make a balance. The general available computational resource of the nodes of set $Y$ is equal to the general required computational resource of the tasks of set $Z$, as follow.

$$
\sum_{a=1}^{h_{y}} \phi_{y_{a}}=\sum_{b=1}^{h_{z}} \phi_{z_{b}}
$$

For this purpose, it is necessary to replace in the corresponding value of node $\left(h_{y}+1\right)$ with the available computational resource $\phi_{y_{h_{y}+1}}$ or instead, replacing the value $h_{z}+1$ related to the task with the required computational resource $\phi_{z_{h_{z}+1}}$, where we have the following equality.

$$
\sum_{a=1}^{h_{y}+1} \phi_{y_{a}}=\sum_{b=1}^{h_{z}+1} \phi_{z_{b}}
$$

In this respect and in order to accept the penalty, it is clear that $s_{z_{h_{z}+1, a}}=0,1 \leq a \leq h_{y}$; $s_{z_{b, h_{y}+1}}=\max _{\substack{1 \leq b \leq h_{z} \\ 1 \leq a \leq h_{y}}} s_{z_{b, a}}, 1 \leq b \leq h_{z}$.

\section{SOLVING THE PROBLEM}

In here, the solving method which performs the search and also provides the breaking for the task sets is step by step stated in which under solving the problem at the network, it permits the network to minimize the average packet delay of network under distributed processing of the tasks.

\subsection{The rule of the minimal penalties}

The basic breaking of the task sets must be done by solving the problem at the network. New subsettings related to the distribution of the nodes based on distributed processing can minimize average packet delay. These subsettings are determined by the matrix $M_{z}^{(\gamma)}$, filled according to the rule of minimal penalties [4] where the element $m_{z_{b, a}}$ determines the computational resource of the node $y_{a} \in Y$, distinguished from others for the subtask processing of the task $z_{b} \in Z$. For developing the basic distribution, a matrix of penalties $S_{z}$ must be created in which the element $s_{z_{b, a}}$ determines a penalty. It will be under selection for the subtask of $z_{b}$ by the node $y_{a}$ (in the unit of computational resource).

The main point related to the minimal penalties is to fill the matrix $M_{z}^{(\gamma)}$ from the element $m_{z_{b, a}}$ in its steps, for which ones are at the matrix $S_{z}$ and also corresponding to the lowest penalty value $s_{z_{b, a}}$. The 
value $\min \left(\phi_{z_{b}}, \phi_{y_{a}}\right)$ is assigned to the element $m_{z_{b, a}}$. The values of the requested computational resource are changed for the task processing $z_{b}$ and the available computational resource of the node $y_{a}$, as below.

$$
\begin{aligned}
& \phi_{z_{b}}=\phi_{z_{b}}-\min \left(\phi_{z_{b}}, \phi_{y_{a}}\right) \\
& \phi_{y_{a}}=\phi_{y_{a}}-\min \left(\phi_{z_{b}}, \phi_{y_{a}}\right)
\end{aligned}
$$

As a further consideration in the matrix $S_{z}$, each row which is corresponding to the request $z_{b}$ is deleted and the necessity in computational resources must be totally satisfied. Similarly, for the column which is corresponding to the node $y_{a}$, the available computational resource is totally used. As a general rule for the column and row, the available computational resource of the node $y_{a}$ is totally used and the necessity in computational resources is satisfied according to the mentioned conditions.

From the rest of elements in matrix $S_{z}$, it is step by step selected again for the elements with the lowest value of the penalty and the process of the distribution of the computational resources which continues until obtaining the requirements (related to all requests of sets $Z$ which will be satisfied for the computational resources). The zero values are also assigned for the unfilled matrix elements $M_{z}^{(\gamma)}$.

\subsection{The potential system}

For the test of the basic distribution in order to optimality, the potential system is built. The potential system can be created only for the non-degenerated distribution plans. The distribution plan $\gamma$ is nondegenerates if the number of the matrix elements $p_{y_{a}}$ is not equal to zero and is equal to $h_{z}+h_{y}-1$ [5]. While creating the basic distribution, the zero elements of matrix $M_{z}^{(\gamma)}$ are less than $h_{z}+h_{y}-1$, namely, the distribution plan $\gamma$ is degenerated.

For each node $y_{a}$, a potential $p_{y_{a}}$ is assigned for each request $z_{b}$ and the potential $p_{z_{b}}$. The potentials $p_{z_{b}}$ and $p_{z_{b}}$ are selected towards each zero element of $M_{z}^{(\gamma)}$. This is for the condition $p_{z_{b}}+p_{y_{a}}=s_{z_{b, a}}$, and for zero element of $M_{z}^{(\gamma)}$, the condition $p_{z_{b}}+p_{y_{a}} \leq s_{z_{b, a}}$ exists. When the number of all potentials is equal to $h_{z}+h_{y}$ (and is not zero), then for the determination of values $p_{z_{b}}$ and $p_{y_{a}}$, there is a solution based on solving the equation system as $p_{z_{b}}+p_{y_{a}}=s_{z_{b, a}}$ with the unknown parameters. For this purpose and under an unknown value, an arbitrary value is assigned. Therefore, the system has the unique solution.

\subsection{The basic estimation}

For each zero element of matrix $M_{z}^{(\gamma)}$, the value of its basic estimation is calculated, which is determined by the difference between the penalty value $s_{z_{b, a}}$ and the potential summary $p_{z_{b}}+p_{y_{a}}$, corresponding to the assigned element. The plan is rational, if the computed values of the basic estimations of $r_{s_{b, a}}$ for all zero elements of $m_{z_{b, a}}$ in the matrix $M_{z}^{(\gamma)}$ will be non-negative, i.e. $r_{s_{b, a}}=s_{z_{b, a}}-\left(p_{z_{b}}+p_{y_{a}}\right) \geq 0$.

If one of the calculated estimations becomes negative, the proposed redistribution of the computational resources is performed. For this purpose, the element $m_{z_{b, a}}$ is selected from the matrix $M_{z}^{(\gamma)}$, in which the basic estimation gets the minimal negative value. For the selected element $m_{z_{b, a}}$ in the matrix $M_{z}^{(\gamma)}$, a closed contour is made. The closed contour represents the consistency of the matrix elements $M_{z}^{(\gamma)}$ and two contiguous items which are located in a same row or in a same column (or the last element which is located in the same row and column as the first). 


\subsection{The rule for the optimizing contour}

The zero element $m_{z_{b, a}}$ of the matrix $M_{z}^{(\gamma)}$, for which $r_{s_{b, a}}$ takes the minimal negative value, is selected by the first element of the closed contour. Other elements of the closed contour are selected from the matrix elements $M_{z}^{(\gamma)}$, and different from the zero.

By having one of the coordinates of the first element as the initial factor, for example, the coordinates of elements in a matrix column of $M_{z}^{(\gamma)}$, the matrix rows of $M_{z}^{(\gamma)}$ are analyzed in this column. The next matrix element of $M_{z}^{(\gamma)}$ in this column, which is different from zero, is selected based on the capacity of the second element of the contour. The third element is searched in the row, in which, it is located the second selected element. If in this row, there is no non-zero element, the second obtained element is deleted from the contour. In the matrix column of $M_{z}^{(\gamma)}$, it is again assigned by the first contour element. And the second zero element is selected to be the second element of the contour. The coordinates of the row of the obtained element indicate the row of the matrix $M_{z}^{(\gamma)}$, in which, the next zero element is selected. The process for searching all the closed contour elements continues till the coordinates of the row of the last obtained element and the first contour element becomes the same.

The interleaved signs are ascribed to the elements of the built closed contour; moreover the positive sign is ascribed to the first contour element. Among the contour elements with the negative sign, the element $m_{z b, a}$ is selected to be the corresponding value for the lowest value. The value of the element $m_{z_{b, a}}$ is added to the values of the elements of the closed contour with the positive sign and is deducted from the value of the elements of the closed contour with the negative sign. As a result, we obtain the new breaking of the task sets, which are used for solving the problem at the network. For the re distribution (new distribution) of $\gamma$, it is built once more, and the system of the potentials calculates the values of the basic estimations for the zero elements $M_{z}^{(\gamma)}$. In the case of the absence of the negative values among the basic estimations for the zero elements at the distribution $\gamma$. So it made a rational decision towards the breaking of the task sets for solving the problem based on the subsettings and distribution.

\section{RESULTS}

The evaluation of effectiveness of the proposed method is performed based on the model of the hyperconverged support system for university E-learning by V. N. Karazin Kharkiv National University. The system boot is simulated during a double-class (90 minutes). It has been considered that the situations have different quantity for the problem in all $\mathrm{N}$-situations simulated. Especially, under $\mathrm{N}=1$, suppose the breakdown of one of the communication channels, and under $\mathrm{N}=10$, suppose the breakdown of the software and hardware.

Evaluation of the quality coefficient for the task distribution vs. the number of the tasks in the process is shown in the Figure 1 where we have the network loading of 30\% in the case of the continuous service. Figure 2 shows the results for the case with the maximum (possible) loading at the network in the case of the continuous service. In both figures, curve "1" shows the classic distribution and curve "2" shows the proposed redistribution. The results of the simulations in Figure 2 clearly show the advantage of the proposed method for the resource redistribution while both software and hardware have been out of service. Table 1 shows the compared methods with some details.

Table 1. The Methods Compared in Simulations

\begin{tabular}{ccc}
\hline Methods & $\begin{array}{c}\text { Related Figures } \\
\text { (Descriptive Results) }\end{array}$ & Notations \\
(Simulated) & Figure 1 & $" 1 "$ \\
Classical & Figure 2 & $" 2 "$ \\
Distribution & Figure 1 & \\
Proposed & Figure 2 & \\
Redistribution &
\end{tabular}




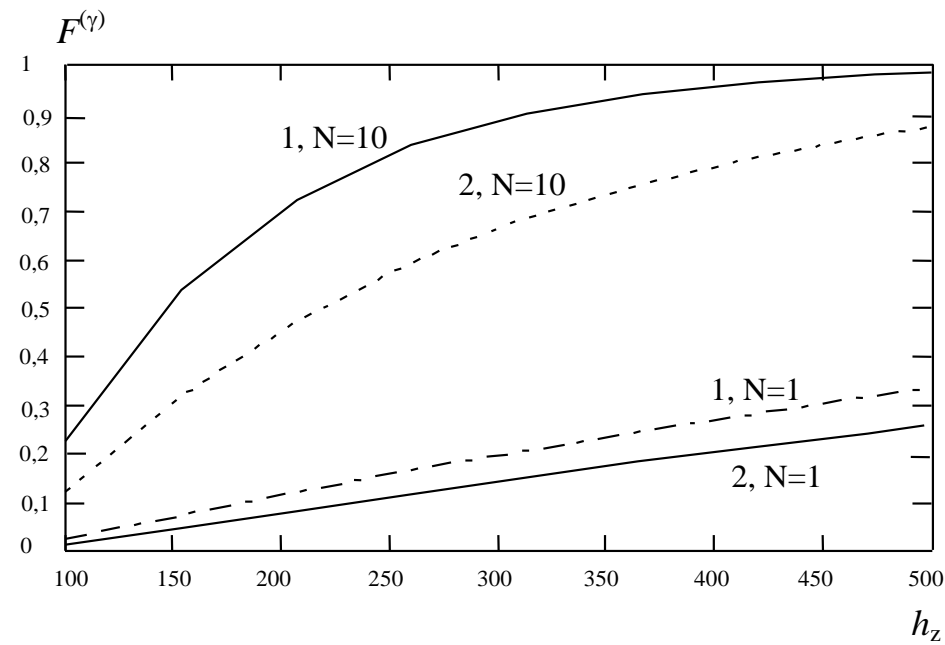

Figure 1. The quality coefficient of the task distribution vs. the number of the tasks (to be processed) at the hyperconverged network under loading of 30\%; "1" shows the classic distribution and "2" shows the proposed redistribution

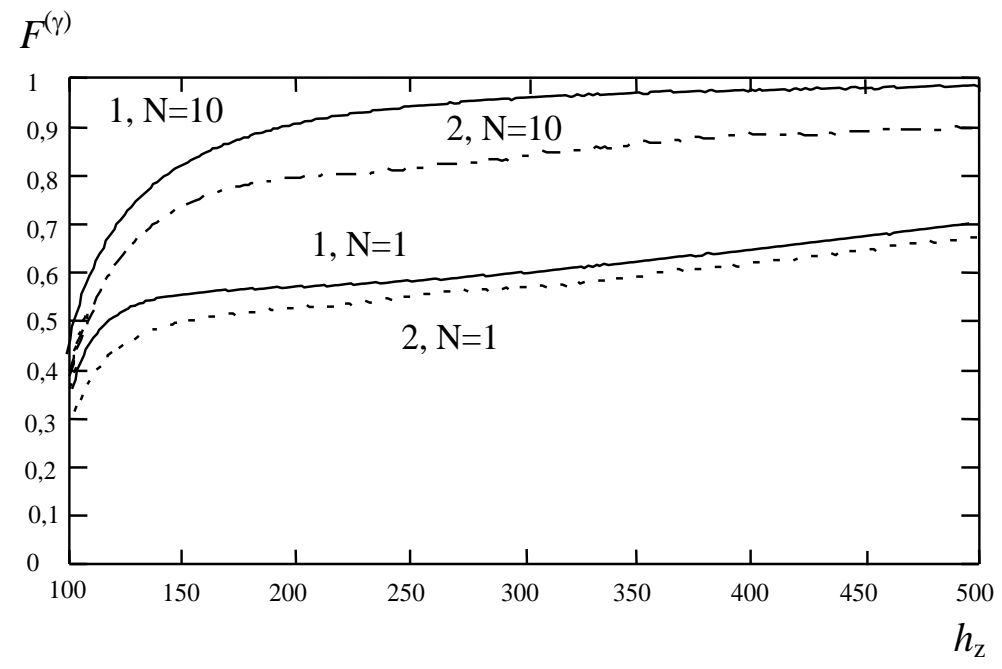

Figure 2. The quality coefficient of the task distribution vs. the number of the tasks (to be processed) at the hyperconverged network under the maximum permitted loading; "1" shows the classic distribution and "2" shows the proposed redistribution

\section{CONCLUSION}

In this paper, a new method for having a better rational task distribution was proposed for hyperconverged networks which it allows us to minimize the average packet delay. The distribution quantity was determined with using a special objective function under the penalties for the cases with the packet delay. The initial redistribution was determined based on the rule of minimal penalties. The obtained task distribution, including the appeared breakdowns, is rational and decreases the average delay of data packet in the hyperconverged network. The evaluation of the effectiveness of the proposed method was performed and we saw that the proposed approach could outperform the classical approach of load distribution. As a future work, this proposed method can be used in other types of networks such as wireless access network [10], MPLS networks [11], ethernet networks [12], wireless sensor networks [13], [14], and all platforms used for 5G-based internet of things (IoT). As another next work, the problem can be resolved based on the proposed formulation but by using other optimization techniques. 


\section{ACKNOWLEDGEMENTS}

The authors would like to thank V.N. Karazin Kharkiv National University for hyperconverged support system model of the university E-learning used in our research. We also thank the managing editor and reviewers for their kind help.

\section{REFERENCES}

[1] Hyper-Converged Edge, Online Documents, Riverbed Ltd., 2017.

[2] https://www.osp.ru/os/2012/04/13015754

[3] http://www.convergedsystem.ru/portfel-produktov-hp-convergedsystem/giperkonvergentnye-sistemy

[4] http://sloanconsortium.org/news_press/january2013_new-study-over-67-million-students-learning-online

[5] K.U. Sri, V. Krishna, "E-Learning: Technological Development in Teaching for school kids", International Journal of Computer Science and Information Technologies, vol. 5, no. 5, pp. 6124-6126, 2014.

[6] G. Kuchuk, A. Kovalenko, V. Kharchenko, A. Shamraev, "Resource-oriented approaches to implementation of traffic control technologies in safety-critical I\&C systems", In book: Green IT Engineering: Components, Network, and Systems Implementation. Springer International Publishing, pp. 313-338, 2017.

[7] S. Fang, Y. Dong, H Shi, "Approximate Modeling of Wireless Channel Based on Service Process Burstiness", Proceedings of the International Conference on Wireless Networks (ICWN), pp. 1-7, 2012.

[8] G. Kuchuk, V. Kharchenko, A. Kovalenko, E. Ruchkov, "Approaches to Selection of Combinatorial Algorithm for Optimization in Network Traffic Control of Safety-Critical Systems", Proceedings of IEEE East-West Design \& Test Symposium (EWDTS'2016), pp. 384-389, 2016.

[9] G.A. Kuchuk, Y.A. Akimova, L.A. Klimenko, "Method of Optimal Allocation of relational Tables", Engineering Simulation, vol. 17, no. 5, pp. 681-689, 2000.

[10] Z. Liu, Y. Shen, Z. Yu, F. Qin, Q. Chen, "Adaptive Resource Allocation Algorithm in Wireless Access Network", TELKOMNIKA (Telecommunication Computing, Electrnonics and Control), vol.14, no. 3, pp. 887-893, 2016.

[11] M. Alhihi, M.R. Khosravi, H. Attar, M. Samour, "Determining the Optimum Number of Paths for Realization of Multi-path Routing in MPLS-TE Networks", TELKOMNIKA (Telecommunication Computing, Electrnonics and Control), vol. 15, no. 4, 2017.

[12] B. Nugraha, B. Fitrianto, F. Bacharuddin, "Mitigating Broadcast Storm on Metro Ethernet Network Using PVST+", TELKOMNIKA (Telecommunication Computing, Electrnonics and Control), vol.14, no. 4, pp. 1559-1564, 2016.

[13] W. Chuan-Yun, Y. Yan, "Research on Topology Control in WSNs Based on Complex Network Model", TELKOMNIKA (Telecommunication Computing, Electrnonics and Control), vol.15, no. 1, pp. 430-437, 2017.

[14] M. R. Khosravi, H. Basri, H. Rostami, "Efficient routing for dense UWSNs with high-speed mobile nodes using spherical divisions", The Journal of Supercomputing, 2017.

\section{BIOGRAPHIES OF AUTHORS}

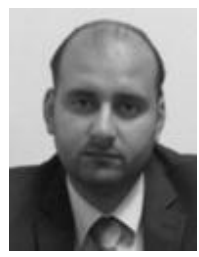

Mohammad Alhihi has received his B.Sc. and M.Sc. in Electrical Engineering (Telecommunications) from National Aerospace University, Ukraine, and the Ph.D. in Telecommunications and Network Engineering from Lvov Polytechnic National University, Ukraine. Since 2012, he has been an Assistant Professor of Electrical Engineering at the Department of Communications and Electronic Engineering, Philadelphia University, Amman, Jordan. His scientific interests include wireless and mobile communications, spread spectrum and MIMO systems, communication networking protocols especially routing and medium access control, traffic engineering and MPLS technology, Cisco systems, information theory and network coding, optimization and soft computing.

Email: malhihi@philadelphia.edu.jo

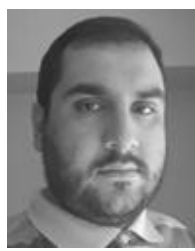

Mohammad R. Khosravi is currently a Senior Researcher for SAR Technology at the Telecommunications Group, Department of Electrical and Electronic Engineering, Shiraz University of Technology, Iran, and also a Research Associate at the Department of Computer Engineering, Persian Gulf University, Iran. He has been an International Committee Member of the conference of IEEE-ICCC 2017, China. In addition, he is currently serving as a Managing Editor of the special sections for Current Medical Imaging Reviews (CMIR), Current Signal Transduction Therapy (CSTT) and International Journal of Sensors, Wireless Communications and Control (IJSWCC). He is an Editorial Board Member/Associate Editor of several international journals in the area of Remote Sensing. His main interests include Statistical Signal and Image Processing, Medical Bioinformatics, Radar Imaging and Satellite Remote Sensing, Computer Communications, Wireless Sensor Networks, Underwater Acoustic Communications, Information Science, Scientometrics, Digital Library Management, Internet Research, Informatics of Scientific Databases, and Citation Analysis.

Email: m.khosravi@ sutech.ac.ir; m.khosravi@mehr.pgu.ac.ir 